\title{
Competencia Digital Docente en el Contexto Paraguayo
}

\section{Digital Competence of the Teacher in the Paraguayan Context}

Delia Lucía Cañete-Estigarribia ${ }^{1}$

\section{อ}

\section{EDICIÓN: \#-CIVTAC}

Recibido: 8/noviembre/2020

Aceptado: 8/febrero/2021

Publicado: 16/abril/2021

我 País

${ }^{1}$ España

\section{IIIII Institución}

${ }^{1}$ Universidad Autónoma de Madrid

\section{\.Correo Eletrónico \\ delia_lucia@hotmail.com}

\section{(iD ORCID}

'https://orcid.org/0000-0001-6544-9739

\section{Citar así: G APA / IEEE}

Cañete-Estigarribia, D. (2021). Competencia Digital Docente en el Contexto Paraguayo. Revista TecnológicaEducativa Docentes 2.0, 11(1), 36-46. https://doi.org/10.37843/rted.v11i1.183

D. Cañete-Estigarribia, "Competencia Digital Docente en el Contexto Paraguayo", RTED, vol. 11, n. ${ }^{\circ} 1$, pp. 3646, abr. 2021.

\section{Resumen}

La sociedad actual es cambiante, esto exige al profesorado utilizar diferentes estrategias con el objetivo de lograr una preparación integral. En este sentido, se considera que la introducción de las Tecnologías de la Información y la Comunicación (TIC) son un factor indispensable para esta preparación integral. Paraguay, al igual que los demás países de Latinoamérica, ha desarrollado planteamientos y propuestas en diferentes ámbitos con el propósito de lograr avanzar en el proceso de incorporación de estas tecnologías en educación, para lo cual es indispensable el desarrollo de competencias digitales. El objetivo de este estudio fue describir qué acciones se han emprendido para el desarrollo de competencias digitales docentes en Paraguay desde el año 2012 hasta el 2020. Se utilizó una metodología de carácter bibliográfico descriptivo, mediante esta se realizó una búsqueda de información en diversas bases de datos, a nivel nacional e internacional. Los resultados evidencian que, si bien Paraguay no es un país en vanguardia con respecto al tema expuesto, se están llevando a cabo esfuerzos para innovar e incorporar esta tecnología en educación mediante programas de capacitación docente a la vez que se dota de esta tecnología a instituciones educativas. No obstante, urge una formación más sólida para el profesorado tanto en los aspectos tecnológicos como en los pedagógicos del quehacer profesional.

Palabras clave: Paraguay, formación docente, competencia digital, innovación.

\section{Abstract}

Today's society is changing; this requires teachers to use different strategies to achieve comprehensive preparation. In this sense, the introduction of Information and Communication Technologies (ICT) is considered an indispensable factor for this thorough preparation. Like the other Latin American countries, Paraguay has developed approaches and proposals in different areas to advance the process of incorporating these technologies in education, for which the development of digital skills is essential. The objective of this study was to describe what actions have been undertaken development of teaching digital competencies in Paraguay from 2012 to 2020. A descriptive bibliographic methodology was used, through which was searched for information in various databases. Data, nationally and internationally. The results show that, although Paraguay is not a country at the forefront concerning the exposed issue, efforts are being made to innovate and incorporate this technology in education through teacher training programs while providing this technology to educational institutions. However, there is an urgent need for more solid training for teachers in the technological and pedagogical aspects of professional work.

Keywords: Gamification, gamification tools, information technologies. 


\section{Introducción}

La sociedad actual es cambiante, esto exige al profesorado utilizar diferentes estrategias con el objetivo de lograr una preparación integral del estudiante. En este sentido, se considera que la introducción de las Tecnologías de la Información y la Comunicación (TIC) en la educación es un factor indispensable, debido a que facilita la adquisición de competencia digital e informacional de los estudiantes (Marqués, 2013). Paraguay, al igual que los demás países de Latinoamérica, ha desarrollado planteamientos y propuestas en diferentes ámbitos con el propósito de lograr avanzar en el proceso de incorporación de estas tecnologías en educación, para lo cual es indispensable el desarrollo de competencias digitales.

Los resultados evidencian que, si bien Paraguay no es un país en vanguardia con respecto al tema expuesto, se están llevando a cabo esfuerzos para innovar e incorporar esta tecnología en educación mediante programas de capacitación docente, a la vez que se dota de esta tecnología a instituciones educativas. No obstante, urge una formación más sólida para el profesorado tanto en los aspectos tecnológicos como en los pedagógicos del quehacer profesional.

El sistema educativo, necesita transformar su esencia asumiendo el compromiso de formar individuos bajo los parámetros de esta nueva era digital, donde el uso de los recursos tecnológicos es fundamental para el progreso social, en el cual, el desarrollo de competencias digitales debe ser considerado un componente fundamental del proceso pedagógico. Sin embargo, la evidencia actual en aula muestra que los jóvenes son expertos en lenguaje digital dominándolo mejor que sus docentes, estableciendo una brecha en competencias digitales entre estudiante y docente (Prensky, 2001). Conforme señala (Consejo Nacional de Educación y Ciencias [CONEC], 2003), los nuevos profesionales en educación inexpertos estas tecnologías, no podrán educar adecuadamente a los estudiantes de este complejo mundo globalizado.

Sumado a ello, se encuentra el hecho que los individuos hacen uso de este recurso sin tener en cuenta el poder potenciador de los procesos cognitivos en aras de la construcción del conocimiento. Estos son los puntos críticos para considerar que deben tener en cuenta al incorporar estas tecnologías en los ambientes pedagógicos. Esta sociedad de hoy en día exige al profesorado implementar diversas estrategias para alcanzar una preparación integral de los individuos mediante estas tecnologías consideradas esenciales (Lion, 2019). En atención a ella, la UNESCO (2013) propone desarrollar nuevas experiencias de aprendizaje centradas en los estudiantes, en que implementen metodologías pedagógicas contextualizadas en la realidad del entorno educativo, así como en el conocimiento de los estudiantes sobre los recursos digitales utilizado para potenciar esta inclusión de nuevas tecnologías en el proceso pedagógico.

De igual manera, contempla estas acciones en los currículos de formación docente, con el propósito de desarrollar competencias digitales de los profesores para una correcta implementación de una práctica educativa sustentada en estas tecnologías. Además, permite crear un puente entre los conocimientos tanto del estudiante como del profesor, promoviendo una co-construcción del conocimiento (Tedesco, 2007). En atención a esta propuesta, en América Latina se pusieron en marcha diversas acciones políticas, económicas e incluso sociales. Sin embargo, la demanda de esta sociedad digital del siglo XXI sigue generando deudas en el área educativa (Ithurburo, 2019). Según Martínez (2013), una de las principales razones es que hasta el momento estas acciones fueron dirigidas más hacia el aspecto de infraestructura, dotación de recursos tecnológicos, transformación educativa.

De igual modo, el autor señala que son los docentes quienes tienen en sus manos la potestad de cambiar esta forma de implementar las tecnologías de información y comunicación para lograr el desarrollo integral del individuo. No obstante, los programas de formación docente implementados en Paraguay no han ofrecido unas adecuadas oportunidades de aprendizaje para optimizar estas adquisiciones de competencias digitales de los profesionales en educación y, por ende, se sigue sin obtener una preparación suficiente para implementar 
metodologías pedagógicas adecuadas permitiendo a los estudiantes aprovechar al máximo estas ventajas que ofrecen estas nuevas tecnologías para el desarrollo del pensamiento crítico, habilidades cognitivas, búsqueda de información, entre otras.

El objetivo que se plantea en el presente estudio es describir qué acciones se han emprendido sobre el desarrollo de competencias digitales de docentes en Paraguay. Para lo cual se desarrollaron tres categorías de análisis, estas comprenden normativa adoptada en torno a estas tecnologías, formación docente con relación al uso de estas, disponibilidad de infraestructura adecuada y de recursos didácticos.

\section{Metodología}

La metodología empleada en el desarrollo de ese estudio teórico consistió en una investigación de carácter bibliográfico descriptivo. De acuerdo con Hernández, Fernández y Baptista (2016) esta investigación busca recoger información de forma independiente sobre los conceptos o variables, así como la realización de una descripción de estos de acuerdo con la información obtenida. Esta investigación fue llevada a cabo a través de una revisión sistemática, bajo el método de Declaración PRISMA el cual consiste en un procedimiento estructurado para cabo una revisión sistemática (Urrútia \& Bonfill, 2010).

En primer lugar, se establecieron las palabras clave: competencia digital; innovación; profesorado; Tecnologías de la Información y la Comunicación; Paraguay. Seguidamente, se procedió a buscar a través en diversas bases de datos, como Google Academic, Dialnet, Scielo, Ministerio de Educación y Ciencias, Paraguay Educa, y catálogos de diversas revistas digitales relacionadas con la temática. Para esta búsqueda se aplicó el sistema booleano AND, aplicando diversas fórmulas de combinación de palabras clave, estas se fueron especificando a medida de la valoración de dicha información siempre sin dejar de lado el contexto paraguayo.

Para priorizar los documentos, se establecieron los siguientes criterios de inclusión, estos son: artículos con fecha de publicación menor a diez (10) años, con texto completo, con información específica del contexto paraguayo al menos en un setenta por ciento $(70 \%)$, documentos con veracidad científica, en idiomas inglés o español con una combinación de al menos dos de las palabras clave por otro lado, a modo de criterios de exclusión se emplearon los siguientes: antigüedad mayor a diez (10) años, que contengan solo resumen, que no presentasen rigurosidad científica o validada. Para reducir los documentos se exportaron al gestor de referencias bibliográficas Zotero donde se aplicaron los criterios de elegibilidad, así como se eliminaros duplicados.

Finalmente, para organizar esta información se fueron filtrando los artículos a través de una lectura rápida elaborándose una tabla en Excel, donde se detallaron los datos relevantes de cada documento (datos bibliográficos, conclusiones, aspectos relevantes de investigación), finalmente se procedió su lectura completa además de elaborar citas textuales, se procedió a estructurar esta información en apartados después de la construcción teórica propuesta de acuerdo con el objetivo de investigación.

\section{Resultados}

Como resultado de la revisión bibliográfica, se identificaron 211 referencias (artículos, capítulos de libros, ponencias, libros, entre otros), de las cuales 149 pertenecen a Google académico, 16 de Dialnet, 18 de Scielo, 12 del MEC, 9 de Paraguay Educa y 7 restantes de otras revistas, y estos fueron analizados con la finalidad de cumplir ciertos criterios de búsqueda relacionados con la temática y eliminar posibles duplicados. Finalmente, se seleccionaron 32 artículos que cumplieron los criterios de inclusión. A continuación, se presentan las tablas representativas de la búsqueda de conceptos en las bases de datos científicas. Es este sentido, conceptos como "Digital Competence of the Teacher" han obtenido los resultados que se resumen en los datos presentados a continuación, como son: total de publicaciones en las que aparece el término, índice $\mathrm{h}$, total de veces citado y número de artículos en los que se cita. 


\section{Gráfico 1}

Datos de búsqueda de Keywords

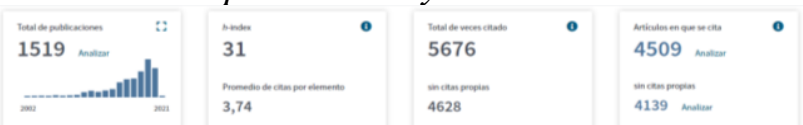

Nota. Resultados de conceptos en las bases de datos científicas, elaboración propia (2020).

\section{Gráfico 2}

Número de citas por año de Keywords

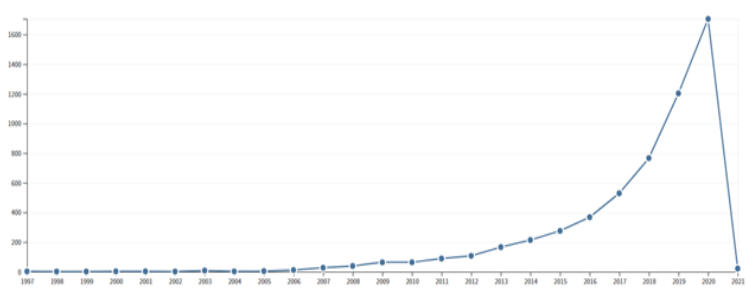

Nota. Resultados por número de citas, elaboración propia (2020).

\section{Principales avances en Paraguay en torno a las TIC}

Se viene reseñando el creciente auge de TIC en esta sociedad actual, esto provoca la implementación de diversas políticas gubernamentales a nivel mundial con el objetivo de asistir a estas demandas en torno a esta nueva era digital. Al respecto, Unesco (2013) reseñó la necesidad de implementar políticas educativas necesarias para el desarrollo de competencias digitales necesarias para enfrentar los embates de esta globalización, en función de ello, la citada organización, a través de diversos tratados, conferencias e investigaciones orienta a los diferentes gobiernos en el establecimiento de medidas facilitadoras del acceso a TIC, así como, medidas educativas en torno a ella (Vezub, 2019).

En América Latina, de acuerdo con el Sistema de Información de Tendencias Educativas (SITEAL), se viene llevando a cabo un importante proceso de integración de TIC a los sistemas educativos, por medio de diversas estrategias de incorporación de estas tecnologías, adquisición de recursos tecnológicos, desarrollo de plataformas digitales, transformaciones curriculares, entre otras. Todo ello demuestra el gran esfuerzo hecho por los estados por implementar políticas públicas para satisfacer necesidades en torno a estas tecnologías (Organización de las Naciones
Unidas, 2019). Sin embargo, esta situación económica en los países de América Latina y el Caribe, caracterizada por índices altos de pobreza, así como de desigualdad social, no permite implementar estas políticas ni lograr su cometido.

Aunque en la actualidad, se siguen implementando acciones dirigidas a lograr una integración curricular, una gestión en escuelas, así como el desarrollo profesional docente. Este desarrollo ocupa un papel de gran importancia, dando origen a programas ambiciosos de formación docente continua (Organización de las Naciones Unidas, 2019). En Paraguay, se estableció una agenda digital con el fin de conocer la transformación del país en este aspecto, así como la mejora de la calidad de vida. En atención a ello, el gobierno ha implementado diversas estrategias con distintos objetivos, entre estos se encuentra: el garantizar un nivel mayor de conectividad, pues hasta el momento apenas alcanza un sesenta por ciento $(60 \%)$.

Con este fin, creó algunas líneas políticas dirigidas a priorizar esta infraestructura. Otro de los objetivos planteados consiste en lograr que cada niño tenga un ordenador, para lo cual se creó el programa "Una computadora por niño" (UCPN). Además, inició investigaciones en robótica (Lion, 2019). La creación del Fondo Nacional de Inversión Pública y Desarrollo (FONACIDE) así como la creación del Fondo para la Excelencia de la Educación y la Investigación (Ley No. 4758) fueron otras de las políticas públicas que el gobierno de Paraguay puso en marcha en torno a la implementación de las TIC. El objetivo principal de esta organización consiste en financiar los programas de incorporación de estas en el sistema educativo, con el propósito de mejorar los procesos educativos, colocando al alcance de cada actor educativo los recursos necesarios para el acceso a estas.

Algunos proyectos virtuales como laboratorios, plataformas, radios o televisiones educativas e incluso enciclopedias cuentan con este apoyo económico. De igual forma, se cuenta con financiación para los programas de apoyo en aras de una mejor calidad de formación docente, aunque para este aspecto solo se dirige un diez por ciento $(10 \%)$ del presupuesto anual del fondo, a pesar de ser considerado uno de los 


\section{EDICIÓN

factores importantes para incorporar estas tecnologías en centros educativos (Ley 4758, artículo 12, Literal b). En el mismo orden de ideas, el Plan Nacional de Educación 2024 (Ministerio de Educación y Cultura, 2011), hizo frente a los desafíos públicos para lograr una educación científico-tecnológica de calidad, propuso transformar los contenidos, objetivos, metodología, programas y currículos, para lograr los cambios con el objetivo de hacer frente a la variedad de necesidades sociales a todos los niveles educacionales.

Asimismo, el Plan Nacional de Desarrollo Paraguay 2014-2030, el Programa País 20192020 así como el Consejo Nacional de Ciencia y Tecnología, a través del plan de Política Nacional de Ciencia, Tecnología e Innovación de Paraguay 2017, establece dentro de sus objetivos para el desarrollo económico-social del país, implementar estrategias para el desarrollo de TIC en el territorio nacional, para contribuir al crecimiento y desarrollo tecnológico.

\section{Formación docente con relación al uso de las TIC}

En cuanto a la normativa que rige la formación docente, Paraguay cuenta en primera instancia con la Ley General de Educación (Ley $\mathrm{N}^{\circ}$ 1264), mediante esta, "garantiza el derecho a aprender, la igualdad de oportunidades de acceder a los conocimientos, a los beneficios de la cultura humanística, de la ciencia y tecnología, sin discriminación alguna (...) así como a la libertad de enseñar, sin más requisitos que idoneidad e integridad" (Artículo 3). Además, establece "programas permanentes de actualización, especialización perfeccionamiento profesional de los educadores" (artículo 132).

De igual manera, se cuenta con el Estatuto del Docente (Ley $\mathrm{N}^{\circ} 1.725 / 01$ ), este en su capítulo VII señala los principios de la formación y actualización permanente estableciendo que los organismos gubernamentales, en coordinación con instituciones educativas responsables, deben apoyar y promover este tipo de procesos.

Asimismo, se encuentra el Reglamento del Estatuto del Educador (Decreto $\mathrm{N}^{\circ} 468$ ), el cual establece que "el Ministerio de Educación y
Cultura (MEC) debe velar por ejecutar actividades de formación enfocadas a docentes nacionales así como estableciendo un impulso a las becas e incluso a los programas de intercambio cultural dirigidos únicamente a educadores que destaquen, con el propósito de que puedan cursar estudios de perfeccionamiento, actualización, especialización, maestrías y/o doctorados".

En cuanto a los programas de formación, cabe destacar el Programa Educador del siglo XXI de la Fundación Gabriel Lewis Galindo, el cual es un programa dirigido a capacitar docentes en ejercicio sobre las TIC (Ministerio de Educación y Cultura, 2011), contribuyendo a construir un nuevo modelo de profesional. Este nuevo modelo del profesional educativo debe responder a lo expuesto en el Plan Nacional de Educación 2024 (Ministerio de Educación y Cultura, 2011) sobre el nuevo perfil de los docentes, donde se considera que las transformaciones vividas tanto en la sociedad cómo y por ende en el sistema educativo, requieren de educadores que asuman un rol de orientador que cuenten además con competencias digitales para educar a través de TIC.

En tal sentido, Robalino \& Korner (2005), señalan que un docente que no maneje tecnologías de la información y comunicación se encuentra en entera desventaja frente a sus estudiantes, apuntan que una política de formación de docentes en TIC "no solo implica apoyar que los docentes conozcan y manejen equipos tecnológicos. Hace falta, sobre todo, contribuir a una reflexión acerca de su impacto en el aprendizaje, su uso adecuado, potencialidades, así como límites" (p. 9).

En concordancia con los anteriores planteamientos, en Paraguay, a pesar de los tropiezos políticos vividos a principios de esta última década, se llevaron a cabo iniciativas para formar a los docentes en TIC, generalmente desarrolladas entre en el Ministerio de Educación y organizaciones civiles, entre ellas, Paraguay Educa. De estas iniciativas hay que destacar:

a) El Programa Ñañemoaranduke, "un proyecto de titulación de maestros bachilleres en escuelas rurales $\mathrm{e}$ indígenas, mediante una plataforma 
virtual implica apropiación, así como el uso de nuevas tecnologías" (PASEM, 2015, p.41);

b) La creación del Centro Tecnológico Serranía en la ciudad de Caacupé, propuesta en 2013, para capacitar a padres y docentes (Paraguay Educa, 2013)

c) El Plan de Capacitación en el Uso de TIC a Docentes de Zonas Rurales, en 2014, el cual se firmó a través de un acuerdo entre la Fundación Iberoamericana y el Ministerio de Educación y Cultura de Paraguay (Paraguay Educa, 2014)

d) El programa "Luces para aprender", de 2015, este consistía en capacitar a docentes en el marco del programa de mejoras de condiciones de aprendizaje incorporando nuevas tecnologías, ejecutado por la Unidad Ejecutora de Programas y Proyectos

e) El Ministerio de Educación y Cultura (Paraguay Educa, 2015), este pretendía una formación en cascada, "apoyada en formación de agentes multiplicadores que más tarde capacitarán a colegas en sus lugares de trabajo" (PASEM, 2015, p.42); implementar este plan a docente rurales pretende robustecer las capacidades técnico-pedagógicas de estos.

f) Además, se implementaron distintos cursos de capacitación: TIC en el Aula de Última Hora Curso Básico de Uso de Herramientas TIC (apoyado por Microsoft Paraguay) y proyecto Nandutí (apoyado por OEI).

A través de los diversos análisis realizados en el marco de los problemas sobre temas docentes de la Oficina Regional de Educación de la Unesco para América Latina y el Caribe (ORALC/Unesco), entre los que se encuentra el de Robalino \& Korner (2005), se evidenció que, aunque se destinaron acciones para avanzar en el perfeccionamiento docente, políticas gubernamentales así como en sistemas educativos no atendieron en plenitud esta demanda, tal y conforme lo señala el PASEM (2015) cuando subraya: "Paraguay aporta un mapa de iniciativas diversas (...) [con] un campo desarticulado con muchas iniciativas efímeras(...) [donde] resulta difícil muchas veces entender cuándo empiezan y cuándo terminan estas acciones, ya que la información es fragmentaria, hay poca sistematización $\mathrm{y}$ monitoreo" (p. 43).

Por tanto, urge una formación más sólida para el profesorado tanto en aspectos tecnológicos como pedagógicos del quehacer profesional, tomando a modo de ejemplo las líneas implementadas por Colombia y Uruguay, reflejando buenos resultados. Para lograr esta formación constante con políticas en materia de TIC, el Ministerio de Educación y Cultura (2014) señala que deben considerarse los pilares de la educación establecidos por la Unesco para el siglo XXI, estos son: "el aprender a conocer, aprender a hacer, aprender a ser, aprender a vivir juntos y aprender a emprender, de manera que le permita llevar a cabo su praxis pedagógica de manera efectiva".

También, el dominio de las competencias digitales, pues, conforme señala Maciel (2017), estas son imprescindibles: debido a las grandes habilidades de los estudiantes en materia digital, el docente no puede optar por improvisar en su accionar educativo, lo que convierte en constante una capacitación siendo además trascendental para el proceso de transformación educativa. En este sentido y en aras de la transformación del proceso pedagógico paraguayo en torno a la integración de TIC en el sistema educativo, Maciel (2017) define las competencias digitales que debe desarrollar el docente del siguiente modo: "la capacidad de convertir datos en información, enseñar no sólo en el sentido crítico para discernir informaciones y conocimientos relevantes, sino conocer los procedimientos para que los educandos aprendan también a producirlos" (p. 7).

Es conveniente considerar que el desarrollo de estas competencias, así como el perfeccionamiento o capacitación docente deben ir de la mano de los avances tecnológicos; por tanto, se debe contar con los recursos digitales necesarios para implementar estas competencias adquiridas ya sea en el uso como en la aplicación de TIC cuando se utilizan a modo de método de enseñanza, para ello el gobierno debe estimar acciones para mejorar la infraestructura digital en las instituciones educativas. De acuerdo con 
los informes de rendición de cuentas presentados por el Ministerio de Educación (2014 - 2019), se ha podido evidenciar que los docentes han recibido la siguiente capacitación en TIC:

a) Durante los años 2012 al 2015, la capacitación fue escasa, se centró sobre todo en formar a los docentes para el programa "Una Computadora Por Niño"; se beneficiaban de esta formación los docentes de instituciones educativas dotadas de este recurso.

b) Para el año 2016, el Ministerio de Educación (2017) reporta la capacitación de 247 docentes de la comunidad de Asunción central en Ciencias, Tecnologías e Innovación Científica; 300 adquirieron una especialización en aprendizaje basado en redes; 100 se instruyeron en formadores de formadores con una especialización en TIC.

c) Para el año 2017 fueron 2200 docentes de Escuelas Básicas Primarias quienes recibieron capacitación a través del curso Aprender Haciendo TIC, continuando con esta especialización en TIC.

d) En el año 2018, se evidencian mayores oportunidades de capacitación a los docentes con los cursos: Plataforma de Aprendizaje Sugar con arreglo a Herramienta Pedagógica; TIC Rupive Nañamoarandu modalidad B-learning; Formación en Plataforma Office 365; Capacitación TIC para Docentes Indígenas; Juguemos Creando 2018; Redes para Docentes de Nivel Medio; y Contenidos TIC.

e) Para el año 2019, el Ministerio de Educación y Ciencia reporta que los docentes recibieron capacitación en TIC Rupive Ñañemoarandu; pensamiento computacional; capacitación docente para pedagogía TIC (360 docentes formados); programación creativa; $\mathrm{y}$ capacitación TIC para el programa 1 a 1. Durante el trascurso de este año 2020, se han realizado cursos sobre trabajo colaborativo en el entorno del Office 365; capacitación en Tutoría Virtual, Educando con tecnologías.

\section{Disponibilidad de infraestructura y recursos digitales}

No se puede negar que el gobierno de Paraguay se encaminó hacia el abastecimiento de recursos digitales para atender necesidades en nuevas tecnologías en instituciones educativas, proceso iniciado en 2012 con la aprobación del Fondo Nacional de Inversión Pública y Desarrollo (FONACIDE) a través de la Ley $\mathrm{N}^{\circ}$ $4758 / 2012$, se destinó el $40 \%$ del fondo fiduciario anual a los proyectos de tecnología educativa (Paraguay Educa, 2013).

Asimismo, el Ministerio de Educación elaboró diversos planes para dotar a instituciones educativas con los recursos digitales necesarios, comenzando en 2012 con el programa "Una Computadora por Niño", incorporado al Plan Director de Tecnología a cargo de la Secretaria de Tecnología, Información y Comunicación, cuyo propósito era brindar conectividad universal a todas las escuelas del país (Paraguay Educa, 2012); el proyecto "Incorporación de Tecnologías de la Información y Comunicación en el Sistema Educativo Nacional", contemplado en el Plan Nacional de Educación 2024, cuyo propósito era: "Dotación de infraestructura digital a instituciones educativas de gestión oficial” (MEC, 2013, p. 1).

El programa "Mejoramiento de las condiciones de aprendizaje mediante la incorporación de Tecnologías de la Información y Comunicación en establecimientos educativos y unidades de gestión educativa en Paraguay", buscando "lograr la construcción, instalación, funcionamiento y certificado de un centro de datos, un portal educativo desarrollado $\mathrm{y}$ funcionando, y una plataforma de aprendizaje en línea incorporada" (MEC, 2017, p.1); más recientemente, el proyecto "Escuelas Conectadas en Paraguay", pretende incorporar TIC en los centros educativos a través del desarrollo por la empresa estatal COPACO de una estructura de fibra óptica para el usufructo de dichos servicios, brindando internet a instituciones educativas. Este tipo de programas plantearon diversos modelos, así como estrategias para la atención de necesidades en materia de equipamiento TIC en instituciones educativas. 
El acceso a Internet es uno de los factores más limitantes: un alto porcentaje de la población no puede acceder a la red, sobre todo en los sectores más pobres, pues el costo de un servicio de banda ancha es alto, de hecho, los estudios estadísticos de Sequera (2020) en su análisis sobre infraestructuras de internet en Paraguay indican que aproximadamente el $81 \%$ de los ciudadanos en edad escolar no cuentan con conexión a internet en sus viviendas. Asimismo, señala incluso aunque se establecieron diversas políticas para fortalecer la educación a través de TIC, mediante los programas antes mencionados, ninguno de los planes propuestos cumplió las metas planificadas al cierre del año 2019, pues apenas lograron un $10 \%$ de cumplimiento.

En tal sentido, las grandes planificaciones hechas durante la última década para avanzar en materia de tecnología en el ámbito pedagógico fueron calificadas de ambiciosas y poco realistas. Por tanto, se insta a tomar medidas más reales que puedan alcanzar un alto índice de cumplimiento (Ithurburu, 2019; Rodolfo, 2014). Mientras tanto, el sistema educativo paraguayo sigue a la cola de los avances en TIC, por lo tanto, conviene que las acciones planteadas puedan satisfacer esta necesidad imperante, a la par de los avances de esta sociedad. Dentro de las metas alcanzadas en equipamiento durante los años 2012 y 2019, el Ministerio de Educación (2014, 2015, 2016, 2017, 2018, 2019), a través de sus informes de rendición de cuentas, junto con Paraguay Educa (2012, 2013, 2014 y 2015), refleja las siguientes:

a) Durante los años 2012, 2013 y 2014, se entregó un gran número de portátiles a los niños de Caacupé a través del proyecto "Una Computadora Por Niño". En el año 2014 se continúa con este proyecto a la vez que se inaugura el Centro Tecnológico Serranía (CTSM) en el Distrito de Caacupé dirigido a ofrecer talleres, así como eventos basados en inclusión digital.

b) El año 2015 fue uno de los más productivos en cuanto a equipamiento de recursos TIC. Se crean 9 salas de informática en diversas instituciones educativas a nivel nacional; se entregaron $3.467 \quad$ computadoras portátiles a los estudiantes de Educación Básica, formando parte del programa "Una Computadora por Niño en Caacupé"; se promovió la conectividad a Internet en 132 instituciones educativas en la Capital y el Departamento Central a través de convenios establecidos entre el MEC - CONATEL y COPACO; se crearon dos aulas digitales cooperando con el Ministerio de Educación de Corea del Sur.

c) En el año 2016, 200 instituciones educativas de diferentes niveles fueron dotadas de servicio de internet por convenio con CONATEL; 3 fueron dotadas con recursos tecnológicos a modo de premio en el programa de concurso de competencias educativas rurales, 2 instituciones recibieron 40 computadoras cada una para crear los laboratorios de informática.

d) Durante los años 2017 y 2018, se continuó con el programa 1 a 1 , entregando portátiles a los estudiantes de diversas instituciones educativas a nivel nacional; se entregaron 4335 notebook a estudiantes de Educación Escolar Básica del distrito de Caacupé; 1 institución consiguió una dotación de una sala completa de informática a través del concurso Súper Vale; se dotaron diversas instituciones educativas con varios recursos tecnológicos, entre ellos: 204 computadoras de escritorio, 1030 notebook, 1049 impresoras, 109 UPS, 95 proyectores; se logró consolidar 782 institutos educativos conectados a internet así como, 25 Institutos de capacitación profesional para docentes con laboratorios informáticos equipados con diversos recursos digitales: pizarras interactivas, ordenadores, impresoras, proyectores; se entregaron 600 notebook a los estudiantes del 2 do y 3 er año de los institutos de formación docente a través del Convenio MEC - COPACO.

e) El año pasado (2019) se entregaron notebooks a los mejores alumnos egresados de bachiller en Alto Paraguay; se continuó con el programa "Una 
Computadora Por Niño"; se logró instalar algunos telecentros. Finalmente, durante este 2020 el MEC se logró entregar laboratorios móviles a diversas instituciones de Bachillerato Técnico en informática.

\section{Discusión}

Para concluir, según la revisión documental en Paraguay, los grandes problemas constatados en cuanto a integración de TIC son: una asignación insuficiente de recursos económicos, una deficiente capacitación de los docentes así como un uso limitado de tecnologías conforme lo expresa la CONEC (2014), aunque se observa que determinadas acciones se siguen poniendo en marcha, la intención es seguir luchando para alcanzar el desarrollo tecnológico del país al mismo tiempo que se potencian los procesos pedagógicos para promover en los estudiantes la construcción del conocimiento, lo esencial es que se potencien los tres pilares claves que se analizaron en este estudio, estos son: normativas adoptadas en Paraguay en torno a TIC, infraestructura, recursos digitales y formación docente.

En cuanto a políticas o Normativas adoptadas en Paraguay en torno a TIC, tomando en cuenta los planteamientos del Sistema de Información de Tendencias Educativas (SITEAL), la Organización de las Naciones Unidas (2019) y Lion (2019), se puede señalar que están bien diseñadas e incluso sustentadas, pues cuentan con una agenda digital estructurada adecuadamente la cual garantiza un mayor nivel de conectividad, priorizar infraestructuras TIC, lograr que cada niño tenga una computadora así como avanzar en robótica, entre otras.

Asimismo, conviene que se lleven a cabo plenamente o se establezcan metas realistas en conformidad con la realidad económico-social de Paraguay, para así lograr los cambios del desarrollo científico-tecnológico deseados, pues, conforme señala Toranzos (en PASEM, 2015), “estas iniciativas son muy recientes y requerirán todavía de muchos esfuerzos técnicos, políticos, económicos e institucionales para comenzar a ofrecer frutos que redunden en prácticas docentes enriquecidas $y$ en docentes protagonistas de un proceso de efectiva transformación educativa" (p.46).

En este sentido, es trascendental la evaluación con mayor profundidad los efectos, así como de las formas de concretar estas propuestas planteadas por el gobierno, con el fin de convertirlas en hechos reales, no sólo en utopías. Para ello, deben buscarse soluciones a esta falta de conectividad, a una calidad deficiente en instituciones educativas, también a una creciente necesidad de dotación de equipos informáticos actualizados; pero sobre todo a la exigencia de implementar un currículo pedagógico en TIC. No basta solo con enseñar a usar computadoras, se debe promover su uso ya que es un potenciador del aprendizaje y la construcción del conocimiento.

Cabe señalar comprender la importancia de la formación en el uso de TIC y la capacitación de los docentes en nuevas metodologías pedagógicas promoviendo la transformación de la práctica educativa a través del uso adecuado de TIC, tal conforme lo refiere Mirete (2010) cuando señala: "la integración de las TIC en el aula requiere un profesorado formado en el uso técnico de las tecnologías, pero sobre todo, en el empleo pedagógico de las mismas" (p. 35); así mismo, cuando afirma: "debería suponer la incorporación de cambios también en la forma de organizar la enseñanza y el aprendizaje, afectando a todas las dimensiones del proceso educativo como la gestión, la comunicación, el currículo" (p. 43).

Estas nuevas formas de enseñanza, actualmente, están centradas en TIC, por tanto, el conocimiento sobre estas permite actuar a la par de demandas actuales de la sociedad en una estrecha relación con los procesos pedagógicos. No obstante, pese a aplicar programas de capacitación docente y dotación tecnológica en instituciones educativas descritas, urge una formación más sólida para el profesorado en los aspectos tecnológicos, así como pedagógicos del quehacer profesional.

Sobre la disponibilidad de infraestructura y recursos digitales, se ha evidenciado desde 2012 con la aprobación del FONACIDE así como con los programas propuestos por el Ministerio de Educación, Paraguay se encaminó a satisfacer demandas tecnológicas en los centros educativos haciendo grandes esfuerzos 
para alcanzar objetivos, no obstante, no han sido suficiente y uno de los factores de mayor demanda insatisfecho es el acceso a Internet pues conforme señala Sequera (2020) un alto porcentaje de la población estudiantil $(81 \%)$ no cuenta con este recurso.

Además, el porcentaje de cumplimiento de los objetivos propuestas en cuanto a dotación apenas alcanza un diez por ciento $(10 \%)$, se sugiere evaluar dichos planteamientos, buscando estrategias para lograr una mayor efectividad. Para culminar, se puede señalar que, si bien Paraguay no es un país en vanguardia con respecto a la temática expuesta, está llevando a cabo grandes esfuerzos para lograr una innovación e incorporación de esta tecnología en educación.

\section{Conclusión}

El objetivo principal de este estudio fue señalar la importancia de la transformación educativa y su importante impacto en el aprendizaje, especialmente en relación con la educación a distancia, los entornos virtuales de enseñanza / aprendizaje y las competencias tecnológicas presente en el profesor y alumno virtual (Barráez, 2020). Es de vital importancia reformar estas competencias desde el conocimiento virtual y el uso de las tecnologías de la información y la comunicación cambiando las practicas docentes y los contenidos.

Se desprende del artículo que el acceso a Internet en Paraguay parte del concepto de brecha digital que, junto con el impacto social del ancho de banda, son determinantes en el aprendizaje de competencias digitales en los profesores. Sin embargo, todo el trabajo no está hecho con el aprendizaje digital y su implementación en aula ya que según (ArribasUrrutia,2019) no es todo y se augura que en un futuro próximo surgirá una nueva brecha digital que se desprende del Big Data.

\section{Referencias}

Arribas-Urrutia, A. (2019). La brecha digital. UNAM.

Barráez, D. P. (2020). La educación a distancia en los procesos educativos: Contribuye significativamente al aprendizaje. Revista Tecnológica-Educativa Docentes 2.0, 8(1), 4149.
CONEC (2003). Situación de la educación en Paraguay. Ediciones y Arte S.R.L.

CONEC (2014). Informe sobre la situación de la educación paraguaya, 2013-2014. Consejo Nacional de Educación y Cultura.

Ithurburu, V. (2019). Políticas digitales en los sistemas educativos de América Latina (2013-2018). Instituto Interamericano de Planeación de la Educación (IIPE) Unesco.

Jiménez, D. F. L. (2006). Realidades comunicativas. Los hemisferios, $9(2), \quad 91-100$. http://dspace.uhemisferios.edu.ec:8080/jspui/handle/123 $456789 / 456$

Leyes Paraguayas (2001). Establece el Estatuto del Educador, Ley $N^{\circ} 1.725 / 01$, Congreso de la Nación Paraguaya, 13 de septiembre de 2001, 5. https://www.bacn.gov.py/archivos/769/20130920114749 .pdf

Leyes Paraguayas (1998). Ley General de Educación, Ley $N^{\circ}$ 1264/1998. Congreso de la Nación Paraguaya, 20 de octubre de 1998, 3. https://www.bacn.gov.py/archivos/3766/2015081708272 8.pdf

Leyes Paraguayas (2012). Crea el Fondo Nacional de Inversión Pública y desarrollo (FONACIDE) y el fondo para la excelencia de la educación y la investigación, Ley $N^{\circ}$ 4758/2012, Congreso de la Nación Paraguaya, Paraguay, 25 de septiembre de 2012, 3. https://www.bacn.gov.py/archivos/3151/2015032409234 6.pdf

Lion, C. (2019). Análisis comparativo de políticas de educación. los desafios y oportunidades de incluir tecnologías en las prácticas educativas. Análisis de casos inspiradores. Instituto Interamericano de Planeación de la Educación (IIPE)- Unesco.

López Jiménez, D. (2004). Consumo de medios en estudiantes de secundaria de Bogotá. Una mirada desde cuatro escuelas de pensamiento de la comunicación. Palabra Clave, (10), 8.

Maciel, M. (2017). La competencia digital del profesorado y su influencia en el uso pedagógico de las tecnologías de información y comunicación en la educación media (Proyecto de Investigación). Universidad Autónoma de Madrid: España.

Marqués, P. (2013). Impacto de las TIC en la educación: funciones y limitaciones. Revista de investigación Editada por Área de Innovación y Desarrollo, 2(1), 1-15. Obtenido https://dialnet.unirioja.es/descarga/articulo/4817326.pdf

Martínez, M. (2013). Reflexiones sobre el profesorado. La formación docente, una realidad postergada. Revista Paraguaya de Educación, 1(3), 19-31.

Ministerio de Educación y Ciencias (MEC) (2017). Programa Mejoramiento de las condiciones de aprendizaje mediante la incorporación de Tecnologías de la Información y Comunicación (TIC) en establecimientos 
educativos y unidades de gestión educativa, en Paraguay. Gobierno Nacional.

Ministerio de Educación y Cultura (MEC) (2013). Incorporación de TIC en el Sistema Educativo Nacional. Ministerio de Educación $\quad \mathrm{y} \quad$ Cultura. https://mec.gov.py/cms_v2/adjuntos/6640

Ministerio de Educación y Ciencia (2014). Informe de Gestión de cuentas 2013-2014. Gobierno Nacional de Paraguay.

Ministerio de Educación y Ciencia (2015). Informe de Rendición de cuentas 2014. Gobierno Nacional de Paraguay.

Ministerio de Educación y Ciencia (2016). Informe de Rendición de cuentas 2015. Gobierno Nacional de Paraguay.

Ministerio de Educación y Ciencia (2017). Informe de Rendición de cuentas 2016. Gobierno Nacional de Paraguay.

Ministerio de Educación y Ciencia (2018). Informe de Rendición de cuentas 2017. Gobierno Nacional de Paraguay.

Ministerio de Educación y Ciencia (2019). Informe de Rendición de cuentas 2018. Gobierno Nacional de Paraguay.

Ministerio de Educación y Ciencia (2020). Informe de Rendición de cuentas 2019. Gobierno Nacional de Paraguay.

Ministerio de Educación y Cultura (2013). Propuesta para la agenda educativa, sintesis de un proceso para su análisis y construcción Fase 1. Juntos por la Educación.

Ministerio de Educación y Cultura (2014). Plan Nacional de Educación 2024. Hacia el centenario de la escuela nueva de Ramón Indalecio Cardozo. Ministerio de Educación y Cultura.

Mirete, A. (2010). Formación docente en TIC. ¿Están los docentes preparados para la (r)evolución TIC? International Journal of Developmental and Educational Psychology, 4(1), 35-44 https://www.redalyc.org/pdf/3498/349832327003.pdf

Mujica-Sequera, R. (2018). Revista Tecnológica-Educativa Docentes 2.0 (RTED). Grupo Docentes 2.0 C.A.

Organización de las Naciones Unidas (2019). Sistema de Información de Tendencias Educativas en América Latina. Instituto Interamericano de Planeación de la Educación (IIPE)- Unesco

Paraguay Educa (2012). Memoria Anual 2012. http://paraguayeduca.org/wp-

content/uploads/2017/06/Memoria-2012-Paraguay-

Educa.pdf

Paraguay Educa (2013). Memoria Anual 2013. http://paraguayeduca.org/wpcontent/uploads/2017/06/Memoria-2013-ParaguayEduca.pdf

Paraguay Educa (2014). Memoria Anual 2014 http://paraguayeduca.org/wpcontent/uploads/2017/06/Memoria-2014-ParaguayEduca.pdf

Paraguay Educa (2015). Memoria Anual 2015 http://paraguayeduca.org/wp-
content/uploads/2017/06/Memoria-2015-ParaguayEduca-.pdf

PASEM - Programa de Apoyo al Sector Educativo del Mercosur. (2015). La incorporación de TIC en la formación docente de los países del MERCOSUR. Teseo.

Programa País 2019-2020: Paraguay. (2019). Paraguay: Organización de Estados Iberoamericanos Para la Educación, la Ciencia y la Cultura Oficina Regional en Paraguay. Gobierno de Paraguay.

Reinoso, J. (9 de enero de 2020). Políticas de desarrollo de las TIC. 5 dias pasión por los negocios. https://www.5dias.com.py/2020/01/politicas-dedesarrollo-de-las-tic/

Robalino, M. y Korner, A. (Coord.). (2005). Formación docente y las tecnologías de información y comunicación. Estudios de casos en Bolivia, Chile, Colombia, Ecuador, México, Panamá, Paraguay y Perú. Unesco.

Rodolfo, E. (2014). El impacto de las TIC en educación: Evidencias de investigaciones y evaluaciones recientes en América Latina. Revista Paraguaya de Educación, 2, 131-150.

Secretaría Técnica de Planificación del Ministerio de Desarrollo Social, Ministerio de Hacienda Política Nacional de Ciencia, Tecnología e Innovación del Paraguay. (2017). Plan Nacional de Desarrollo Paraguay 2014-2030. Consejo Nacional de Ciencia y Tecnología.

Sequera, M. (2020). La educación virtual y la infraestructura de internet en Paraguay. Lugar de publicación: TECDIC. Paraguay: Asociación de Tecnología, Educación, Desarrollo, Investigación y Comunicación. https://www.tedic.org/la-educacion-virtual-y-lainfraestructura-de-internet-en-paraguay/

Tedesco, J. (2007). Las TIC en la agenda de la política educativa, en Las TIC: del aula a la agenda política. IIPE Unesco y UNICEF Argentina.

Unesco (2013). Enfoques estratégicos sobre las TIC en educación en América Latina y el Caribe. Oficina Regional de Educación para América Latina y el Caribe (OREAL/Unesco)

Vezub, L. (2019). Análisis Comparativos de Politicas de Educación. Las politicas de Formación Docente Continua en América Latina. Mapeo exploratorio en 13 países. SITEAL - IIPE Unesco. 